\title{
Avaliação da Qualidade de Vida de Portadores de Leucemia Mieloide Crônica em João Pessoa-PB no período de 2015 a 2016
}

\author{
Evaluación de la calidad de vida de pacientes con leucemia mieloide crónica en João \\ Pessoa-PB de 2015 a 2016
}

\author{
Quality of Life Assessment of Patients with Chronic Myeloid Leukemia in João Pessoa-PB \\ from 2015 to 2016
}

\begin{abstract}
Patrícia Maria Simões de Albuquerque ${ }^{1 *}$, Daniele Idalino Janebro Ximenes ${ }^{1}$, Margareth de Fátima Formiga Melo Diniz'.
\end{abstract}

\section{RESUMO}

Objetivo: Avaliar a qualidade de vida do paciente com Leucemia Mieloide Crônica (LMC) submetido à quimioterapia oral. Métodos: Para coleta de dados utilizou-se pesquisa quantitativa a partir da aplicação de dois instrumentos: o de dados sócio-demográficos, clínicos e terapêuticos; e o contendo o questionário World Health Organization Quality of Life (WHOQOL-bref), para avaliação da qualidade de vida. A amostra foi constituída por 105 pacientes atendidos no ambulatório de quimioterapia de um hospital de João Pessoa-PB, no período de setembro de 2015 a setembro de 2016. Resultados: Foi evidenciada a prevalência do sexo masculino e a média de idade dos pacientes foi de 31 a 40 anos. De acordo com os resultados encontrados, pode-se verificar que os domínios físicos e psicológicos foram os mais afetados, com diferença estatisticamente significativa para o domínio físico em relação a todos os domínios. É visto que a neoplasia hematológica causa mudanças na vida do paciente, demonstrando que as reações adversas ao tratamento influenciam negativamente na qualidade de vida dos mesmos. Conclusão: Assim sendo, verifica-se a necessidade do cuidado dos profissionais da saúde no impacto que o tratamento provoca nesse processo do paciente com LMC, levando em consideração os domínios de qualidade de vida afetados.

Palavras-chave: Qualidade de Vida, Leucemia Mieloide Crônica, Quimioterapia, Neoplasia hematológica.

\begin{abstract}
Objective: To evaluate the quality of life of the patient with Chronic Myeloid Leukemia (CML) submitted to oral chemotherapy. Methods: Quantitative research was used to collect data from the application of two instruments: socio-demographic, clinical and therapeutic data; and the World Health Organization Quality of Life (WHOQOL-bref) questionnaire to assess quality of life. The sample consisted of 105 patients seen at the chemotherapy outpatient clinic of a hospital in João Pessoa-PB, from September 2015 to September 2016. Results: It was evidenced the prevalence of males and the mean age of the patients was 31 to 40 years. According to the results found, it can be verified that the physical and psychological domains were the most affected, with a statistically significant difference for the physical domain in relation to all the domains. It is seen that hematologic neoplasia causes changes in the patient's life, demonstrating that adverse reactions to treatment negatively influence their quality of life. Conclusion: Therefore, it is necessary to take care of the health professionals in the impact that the treatment causes in this process of the patient with CML, taking into account the domains of quality of life affected.
\end{abstract}

Key words: Quality of Life, Chronic Myeloid Leukemia, Chemotherapy.

${ }^{1}$ Universidade Federal da Paraíba (UFPB), João Pessoa-Paraíba *E-mail: simoespatricia@hotmail.com

SUBMETIDO EM: 7/2019

ACEITO EM: 7/2019

PUBLICADO EM: 8/2019 


\section{RESUMEN}

Objetivo: Evaluar la calidad de vida del paciente con Leucemia Mieloide Crónica sometido a la quimioterapia oral. Métodos: Se realizó investigación cantitativa a partir de aplicación del cuestionario genérico WHOQOLbref. La muestra fue compuesta de 105 pacientes del sector de ambulatorio de quimioterapia de un hospital del municipio de João Pessoa-PB, en el período de septembre de 2015 a septembre de 2016. Resultados: Fue evidenciada la prevalencia del sexo masculino y la media de edad de los participantes estuvo entre 31 a 40 años. De acuerdo con los resultados encontrados, se puede inferir que los dominios físicos y psicológicos fueron los que sufrieron más alteración, pero con diferenciación estadísticamente significativa para el dominio físico en relación a todos dominios. Se señala que la neoplasia hematológica altera prácticamente todos aspectos de la vida del individuo y causa profundas alteraciones en su rutina y hábitos de vida demostrando que las reacciones adversas al tratamiento influyeron negativamente en la calidad de vida de los pacientes. Conclusión: Siendo, es vista la necesidad y la importancia del cuidado de los profesionales de salud en la intervención de dicho proceso, consideración los dominios de Calidad de Vida Relacionada a la Salud de los afectados.

Palabras Ilaves: Calidad de Vida, Leucemia Mieloide Crónica, Quimioterapia.

\section{INTRODUÇÃO}

A Leucemia Mieloide Crônica (LMC) é uma doença mieloproliferativa clonal, que corresponde a 15-20\% das leucemias, com prevalência estimada de 1,0 a 1,5 casos por 100.000 habitantes (VARDIMAN JW et al., 2009). A doença afeta preferencialmente homens em relação às mulheres, numa proporção $2: 1$, sua incidência aumenta com a idade sendo os indivíduos mais afetados entre 45 e 55 anos com pico de incidência aos 53 anos (BORTOLHEIRO TC e CHIATTONE CS, 2008; CAMPOS MGVC. et al., 2010; CHAUFFAILLE ML, 2010; FRAZER R e IRVINE AE, 2007). Pesquisas brasileiras indicam que a mediana de idade na apresentação da doença é de 40 a 46 anos, sendo assim, dez anos a menos que os valores de 55 e 66 anos encontrados, respectivamente, na Europa e Estados Unidos, na literatura internacional (BENEDIT I, et al., 2012; SILVEIRA CA e FERRARI I, 2012).

O início do tratamento com o uso dos inibidores tirosina quinase (TKI) desde 2001 representaram um importante avanço no tratamento da LMC, sendo possível obter sobrevida praticamente similar à da população geral. Embora que estes TKIs condicionam os eventos adversos, considerados um dos maiores estigmas associados à doença (BORTOLHEIRO TC e CHIATTONE CS, 2008; CAMPOS MGVC et al., 2010; LANEUVILLE P, 2018).

O tratamento com o uso dos TKIs na LMC modificou a história natural da doença, é um exemplo de revolução terapêutica devido à alta eficácia do tratamento e como se trata uma doença crônica com o uso de TKIs por longos períodos; deve-se dar importância ao manejo dos eventos adversos, através da sua prevenção ou minimização, permitindo, portanto, um incremento na qualidade de vida dos pacientes (ROSS DM, 2018).

Dada à complexidade do tratamento com os medicamentos antineoplásicos, o que leva a diferentes demandas físicas e psíquicas, afligindo os pacientes, é vista a necessidade de mais atenção em relação à qualidade de vida dos mesmos ( $\mathrm{YU} \mathrm{CH}$, et al. 2017).

$\mathrm{Na}$ área da saúde, o conceito de qualidade de vida $(\mathrm{QV})$ é relativamente recente e, nos últimos anos, tem sofrido influência de novos paradigmas. O que determina o processo saúde ser caracterizado como multifatorial e complexo, é ser relacionado aos aspectos econômicos, sócio-culturais, à experiência pessoal e a estilos de vida (SEIDL EMF e ZANNON CMLC, 2004). Pela Organização Mundial de Saúde (OMS) qualidade de vida é definida como "a percepção do indivíduo de sua posição na vida no contexto da cultura e sistema de valores nos quais ele vive e em relação aos seus objetivos, expectativas, padrões e preocupações" (WORLD, 2009). 
$\mathrm{Na}$ área da oncologia devido à cronicidade do estado de saúde interferir na vida cotidiana do paciente, a qualidade de vida está associada à subjetividade do individuo na percepção comparativa com que ele associa o conceito do que é possível do ideal (MICHELONE APC e SANTOS VLCG, 2004).

Desta forma, o presente estudo teve como objetivo analisar os impactos positivos e negativos sobre a qualidade de vida dos pacientes portadores de Leucemia Mieloide Crônica (LMC) em tratamento quimioterápico com inibidores de tirosina-quinase (TKIs).

\section{MÉTODOS}

Trata-se de uma pesquisa quantitativa, com delineamento do tipo transversal, desenvolvida no Serviço de oncohematologia do Hospital Napoleão Laureano (HNL) mantido pela Fundação Napoleão Laureano sem fins lucrativos, localizado na cidade de João Pessoa, na Paraíba, Brasil, no período de setembro de 2015 a setembro de 2016.

Os participantes do estudo foram pacientes portadores de Leucemia Mieloide Crônica (LMC) Filadélfia positivo submetidos a tratamento quimioterápico oral com inibidores de tirosina-quinase (TKIs): imatinibe, dasatinibe e nilotinibe.

A população foi composta de 105 pacientes que tiveram como critérios de inclusão: adulto de ambos os sexos acima de 18 anos, com diagnóstico de LMC, estar realizando tratamento quimioterápico oral no Ambulatório de oncohematologia e apresentar condições para responder os questionamentos. Após a inclusão na pesquisa, todos os pacientes foram submetidos à avaliação no Ambulatório de oncohematologia, durante a consulta, na aplicação dos instrumentos do estudo.

Para tornar exeqüíveis os objetivos propostos, foi utilizado um formulário contendo duas partes para a coleta de dados: a primeira, continha os dados sócio-demográficos, clínicos e terapêuticos; e a segunda parte, contendo o questionário World Health Organization Quality of Life (WHOQOL-bref), para avaliação da qualidade de vida (THE WHOQOL, 1998).

Este instrumento validado no Brasil (FLECK MPA, 2000), apresenta 26 questões, sendo duas gerais e as demais compostas por quatro domínios: físico, psicológico, relações sociais e meio ambiente, os quais são representados por 24 facetas que compõem o instrumento original. Cada item é composto por cinco graus de intensidades e o paciente escolhe uma delas, valendo cada alternativa pontos diferentes.

Foi realizada uma análise estatística descritiva dos dados pelo programa estatístico Statistical Package for Social Sciences (SPSS), versão 21, seguindo as recomendações da OMS para avaliação das respostas do questionário de qualidade de vida WHOQOL-bref, assim como para a análise estatística.

Foram utilizados testes inferenciais paramétricos One Way ANOVA com teste posto hoc de bonferroni, teste $t$ de Studant, correlação de Pearson e regressão linear com método stepwise para verificar a relação dos domínios do WOOQOL-bref com as medidas relacionadas à doença e para tanto foi aceito uma significância estatística menor ou igual a 0,05, ou seja, $p \leq 0,05$.

No presente estudo, os participantes foram incluídos de acordo com os procedimentos éticos exigidos pela Resolução №466/12 versão 2012a do Conselho Nacional de Saúde, aos quais foram explicados os objetivos da pesquisa e o caráter voluntário da mesma e solicitada a assinatura do Termo de Consentimento Livre e Esclarecido, de forma a garantir o anonimato e a confidencialidade dos dados. O projeto foi submetido ao Comitê de Ética em Pesquisa do Centro de Ciências da Saúde, da Universidade Federal da Paraíba CEP/CSS, sob Prot. ํo 0189/13 e CAAE: 14025313.6.0000.5188.

\section{RESULTADOS}

A tabela 1 mostra que a amostra foi composta de maioria do sexo masculino, de cor parda, casados, com ensino fundamental incompleto, com trabalho remunerado, recebendo um salário mínimo, com residência própria e de religião católica. 
Tabela 1- Descrição dos dados demográficos da amostra. João Pessoa, Paraíba, 2015.

\begin{tabular}{|c|c|c|}
\hline & $\mathbf{n}$ & $\%$ \\
\hline \multicolumn{3}{|l|}{ Gênero } \\
\hline Feminino & 44 & 42,3 \\
\hline Masculino & 60 & 57,7 \\
\hline \multicolumn{3}{|l|}{ Raça ou grupo étnico } \\
\hline Branca & 35 & 34,0 \\
\hline Negra & 5 & 4,9 \\
\hline Parda & 63 & 61,2 \\
\hline \multicolumn{3}{|l|}{ Estado civil } \\
\hline Solteiro & 32 & 32,0 \\
\hline Casado & 55 & 55,0 \\
\hline Separado & 7 & 7,0 \\
\hline Viúvo & 6 & 6,0 \\
\hline \multicolumn{3}{|l|}{ Escolaridade } \\
\hline Analfabeto & 12 & 12,8 \\
\hline Fundamental incompleto & 46 & 48,9 \\
\hline Fundamental completo & 10 & 10,6 \\
\hline Ensino médio incompleto & 5 & 5,3 \\
\hline Ensino médio completo & 20 & 21,3 \\
\hline Superior & 1 & 1,1 \\
\hline \multicolumn{3}{|l|}{ Ocupação } \\
\hline Trabalhador Remunerado & 34 & 36,2 \\
\hline Do Lar & 16 & 17,0 \\
\hline Agricultor & 25 & 26,6 \\
\hline Estudante & 8 & 8,5 \\
\hline Aposentado & 7 & 7,4 \\
\hline INSS & 3 & 3,2 \\
\hline Outros & 1 & 1,1 \\
\hline \multicolumn{3}{|l|}{ Renda familiar } \\
\hline Menos de um salário mínimo & 1 & 2,1 \\
\hline Um Salário Mínimo & 43 & 89,6 \\
\hline Dois Salários Mínimos & 3 & 6,3 \\
\hline Três a Cinco Salários Mínimos & 1 & 2,1 \\
\hline \multicolumn{3}{|l|}{ Moradia } \\
\hline Própria & 73 & 82,0 \\
\hline Alugada & 10 & 11,2 \\
\hline Emprestada & 4 & 4,5 \\
\hline Mora com a família ou amigos & 2 & 2,2 \\
\hline \multicolumn{3}{|l|}{ Religião } \\
\hline Católica & 81 & 77,9 \\
\hline Evangélica & 10 & 9,6 \\
\hline Não Informado & 13 & 12,5 \\
\hline
\end{tabular}

* Existiram casos omissos para as variáveis

Fonte: Dados da pesquisa, 2015.

A tabela 2 faz uma descrição dos dados relacionados à saúde e aos hábitos de vida. As idades diagnosticas foram variadas, sendo grupo mais prevalente entre 31 a 40 anos e quase metade da amostra fica a menos de $100 \mathrm{Km}$ de distância do ambulatório. A maioria declarou não fumar, não beber bebidas alcoólicas e nem possuir outras doenças. 
Tabela 2 - Descrição dos dados de saúde e hábitos de vida. João Pessoa, Paraíba, 2015.

\begin{tabular}{|c|c|c|}
\hline & $\mathbf{n}$ & $\%$ \\
\hline \multicolumn{3}{|l|}{ Idade ao diagnóstico } \\
\hline 18 a 30 & 18 & 17,3 \\
\hline 31 a 40 & 24 & 23,1 \\
\hline 41 a 50 & 19 & 18,3 \\
\hline 51 a 60 & 15 & 14,4 \\
\hline \multicolumn{3}{|c|}{ Distância entre moradia e ambulatório especializado } \\
\hline$<100 \mathrm{~km}$ & 46 & 44,2 \\
\hline $101-200 \mathrm{~km}$ & 27 & 26,0 \\
\hline $201-300$ km & 9 & 8,7 \\
\hline $301-400 \mathrm{~km}$ & 10 & 9,6 \\
\hline $401-500 \mathrm{~km}$ & 12 & 11,5 \\
\hline \multicolumn{3}{|l|}{ Fuma } \\
\hline Sim & 8 & 14,8 \\
\hline Não & 27 & 50,0 \\
\hline Já fumei, mas parei & 19 & 35,2 \\
\hline \multicolumn{3}{|l|}{ Faz uso de Bebida Alcoólica } \\
\hline Sim & 8 & 17,0 \\
\hline Não & 31 & 66,0 \\
\hline Já bebi, mas parei & 8 & 17,0 \\
\hline \multicolumn{3}{|l|}{ Possui outra doença } \\
\hline Doenças cardiopatas & 13 & 25,0 \\
\hline Diabetes & 4 & 7,7 \\
\hline Gastro intestinal & 3 & 5,8 \\
\hline Outro câncer & 2 & 3,8 \\
\hline Outras doenças, exceto câncer & 4 & 7,7 \\
\hline Não Possui Doença & 26 & 50,0 \\
\hline
\end{tabular}

${ }^{*}$ Existiram casos omissos para as variáveis

Fonte:: Dados da pesquisa, 2015.

A tabela 3 apresenta e faz uma comparação das médias entre os domínios de qualidade de vida. Os resultados mostram que as maiores médias foram as dos domínios psicológico e social, com diferenças estatisticamente significativas dos domínios físico e ambiental. O domínio físico foi o que apresentou a pior média, com diferença estatisticamente significativa de todos os outros domínios.

Tabela 3 - Comparações entre as médias de dos domínios de qualidade de vida. João Pessoa, Paraíba, 2015.

\begin{tabular}{lcccc}
\hline & Média & Desvio padrão & Mínimo & Máximo \\
\hline QV física & $56,33^{\mathbf{a}}$ & 17,83 & 10,71 & 89,29 \\
\hline QV psicológico & $69,57^{\mathbf{b}}$ & 15,1 & 29,17 & 100 \\
\hline QV social & $69,73^{\mathbf{b}}$ & 16,51 & 16,67 & 100 \\
\hline QV ambiental & $61,47^{\mathbf{c}}$ & 12,74 & 31,25 & 93,75 \\
\hline QV geral & 69,3 & 16,72 & 12,5 & 100 \\
\hline
\end{tabular}

- [Wilks Lambda $(F)=23,48 ; p \leq 0,01]$

- Obs. Letras diferentes entre as médias indicam diferenças estatisticamente significativas $(p \leq 0,05)$.

Fonte: Dados da pesquisa, 2015. 


\section{DISCUSSÃO}

De acordo com os dados do INCA (2017), a incidência do câncer ocorre predominantemente, em população com baixo nível de escolaridade e na faixa etária da idade adulta, a qual é compatível com indivíduos trabalhadores, sendo estes detectados nesta pesquisa. Esses dados são característicos desse tipo de neoplasia hematológica, tanto ao predomínio do sexo quanto à idade (BENEDIT I et al.,2012; SILVA ABA. et al., 2016; SILVEIRA CA e FERRARI I, 2012). Dando sequência, quando nos referimos à qualidade de vida um dado investigado importante é o fato de que quase metade da amostra fica a menos de $100 \mathrm{Km}$ de distância do ambulatório, o que influência positivamente, na QV desses pacientes, uma vez que fica mais fácil a ida ao hospital.

A clínica do portador de câncer já favorece naturalmente o isolamento social dos mesmos e somado também ao fator preponderante do tempo prolongado de tratamento, que no caso da população estudada, acaba se tornando um importante item a ser destacado, uma vez que se trata de uma doença crônica. Desta forma, morar pouco distante do centro de tratamento pode ter contribuído como ponto positivo, interferindo diretamente na melhora da qualidade de vida e no percurso terapêutico. Neste sentido, o convívio com familiares e amigos que estimulam a vivência de atividades cotidianas é fundamental para influenciar na melhora da QV. Contrariamente, o estudo de Calefi KAC (2014) mostraram que a maior parte dos pacientes com neoplasia hematológica submetido a tratamento quimioterápico morava fora da região onde eram tratados o que influenciou negativamente, na QV desses pacientes, uma vez que ficava mais difícil a convivência com a família e a visitação de amigos e pessoas próximas do seu cotidiano.

Dentro dos parâmetros mais significativos, frente às análises realizadas com pacientes com câncer submetidos ao tratamento quimioterápico, o processo terapêutico remete a uma variedade de reações adversas que causam desconforto. A dor está entre os mais presentes efeitos adversos da quimioterapia, a qual apresenta um forte impacto na QV (USUKI, K. et al, 2012; IRVINE E e WIILLAMS C, 2013; SILVA ABA. et al., 2016).

O domínio físico, com a aplicação do instrumento de avaliação de Qualidade de Vida, WHOQOL-bref, se destacou como o mais alterado e tem relação com: dor e desconforto, energia e fadiga, sono e repouso, mobilidade, atividade da vida cotidiana, dependência de medicações e tratamento e capacidade para 0 trabalho, assim como visto também por Calefi KAC (2014), em sua pesquisa com qualidade de vida nos portadores de câncer hematológico.

Os resultados demonstrados para o domínio físico, durante o estudo, podem ter sido afetados pela limitação do diagnóstico da neoplasia hematológica, pelos efeitos adversos com o uso dos inibidores de tirosina-quinase e algumas comorbidades apresentadas, como diabetes, doenças cardiopatas e gastrointestinal. Estes fatores levam a fadiga pelo tempo prolongado de tratamento, utilização de medicações tóxicas ao organismo, além de que algumas vezes, geram restrições e/ou afastamento do trabalho. Em relação aos resultados do domínio físico, destaca-se que a fadiga (60\%) foi um dos sintomas que mais alterou a QV do paciente nesse estudo, corroborando com outra pesquisa de pacientes com câncer hematológico, realizado por Andrade V, et al. (2013), onde a fadiga apresentou média alta (65,57\%).

Um estudo realizado no ambulatório da Associação de Combate ao Câncer no Brasil Central, por Vena C, et al. (2004), observou que a fadiga relacionada a neoplasias é um problema clínico com uma abordagem complexa, multifatorial e multidimensional, uma vez que ocorrem dificuldades na determinação de um método padrão para mensurar a fadiga relacionada a doença neoplásica, tornando complicado determinar a forma de comparar os resultados dos achados das pesquisas.

A avaliação da qualidade de vida tem sido considerada importante quando se avalia os resultados de tratamento antineoplásico. A medida da mesma tem mostrado verdadeiro impacto dessa terapêutica não só na sensação de bem-estar físico, mas também no estado emocional dos pacientes. O domínio psicológico também foi afetado neste estudo, o qual é composto pelos itens: sentimentos positivos e negativos, memória e concentração, imagem corporal, aparência e crenças. A alteração desse domínio pode ter se dado pelo próprio diagnóstico, assim como pela utilização de quimioterápicos no tratamento (ANDRADE V, et al., 2013). 
Ainda em relação às preocupações vistas pela amostra estudada, foram evidenciados alguns problemas de autoimagem que possivelmente estiveram relacionados ao evento alopecia e também perda de peso. Os fatores interligados a autoimagem são relevantes na avaliação da qualidade de vida e também foi visto no estudo de Terra FS et al. (2013) com pacientes oncológicos submetidos à quimioterapia. A alteração da imagem corporal faz com que os pacientes adquiram sentimentos negativos em relação à sua aparência $\mathrm{e}$ muitas vezes acabam se isolando do convívio social, o que acarreta alterações na sua rotina e hábitos de vida devido, principalmente, a diminuição da autoestima. Estes fatos vistos no nosso estudo também estiveram presentes no trabalho de Chaves PL (2010) com pacientes em uso de quimioterapia ambulatorial.

As neoplasias hematológicas com seus tratamentos geram muitas mudanças na aparência e organismo do individuo, levando a depressão nos mais vulneráveis, o que foi associado a uma menor qualidade de vida no estudo de $\mathrm{Yu} \mathrm{CH}$ et al. (2017). Os resultados apresentados em nosso estudo endossam também tal relação, visto que a preocupação com a imagem causa ansiedade e estresse afetando o domínio psicológico.

Acredita-se que devido à escassez de dados prospectivos, mais pesquisas devem ocorrer para avaliar o valor prognóstico da quimioterapia antineoplásica na qualidade de vida de pacientes onco-hematológicos, em especial aqueles que apresentam estado crônico longo como os portadores de leucemia Mieloide crônica, que requer persistência durante o tratamento.

\section{CONCLUSÃO}

Pelos dados apresentados, pode-se concluir que os baixos escores do domínio físico tem ocasionado alterações na qualidade de vida dos pacientes com leucemia Mieloide crônica, devido à limitação da própria doença e aos efeitos do tratamento da mesma. É necessário prestar assistência de qualidade a essa categoria de pacientes, para tanto é relevante uma equipe multiprofissional de saúde que apresente conhecimento fisiológico da doença, dos tipos de tratamento e do impacto que estes acarretam na vida dos indivíduos com a doença e dos seus familiares de forma a detectar, intervir e avaliar os sinais e sintomas da doença e as reações adversas dos medicamentos utilizados no tratamento, visando a uma melhor qualidade de vida relacionada à saúde dos mesmos.

\section{REFERÊNCIAS}

1. ANDRADE V, et al., SAWADA NO, BARICHELLO E. Qualidade de vida de pacientes com câncer hematológico em tratamento quimioterápico. Rev Esc Enferm USP, 2013:47(2):355-361.

2. BENEDIT I et al., Evaluation of Long-Term Outcome, Cytogenetic and Molecular Responses with Imatinib Mesylate in Early and Late Chronic -Phase Chronic Myeloid in Early and Late Chronic-Phase Chronic Myeloid Leukemia: a report from a Singe Institute. Acta Haematol, 2012: 128(10):223-2322.

3. BORTOLHEIRO TC, CHIATTONE CS. Leucemia Mieloide Crônica: história natural e classificação. Rev.bras. hematol. Hemoter, 2008: 30(Supl. 1): 3-7.

4. BRASIL. Ministério da Saúde. Instituto Nacional de Câncer. Estimativas 2018: incidência de câncer no Brasil. Coordenação de Prevenção e Vigilância. Rio de Janeiro: INCA, 2017.

5. CALEFI KAC. Qualidade de vida do paciente com neoplasia hematológica submetido à quimioterapia. Rev Min Enferm., 2014: 18(1): 41-47.

6. CAMPOS MGVC, et al. Chronic myeloid leukemia : A disease of youth in Brazil. Leukemia Research, 2010: 34:.542544.

7. CHAUFFAILLE ML. Neoplasias mieloproliferativas: revisão dos critérios diagnósticos e dos aspectos clínicos. Revista brasileira de hematologiae hemoterapia. [online], 2010:32 (4): 38-311516-6.

8. CHAVES PL. Avaliação da qualidade de vida do paciente com câncer colorretal em quimioterapia ambulatorial. Dissertação (Programa de Pós-Graduação em Enfermagem) - Escola de Enfermagem Porto Alegre: Universidade Federal do Rio Grande do Sul, 2010.

9. FLECK MPA, et al. Aplicação da versão em português do instrumento abreviado de avaliação da qualidade de vida "WHOQOL-bref". Rev Saúde Pública, 2000; 34(2):178-83.

10. FRAZER R, IRVINE AE, McMULLIN MF. Chronic myeloid Leukaemia in the 21st Century.Ulster Medical Journal, 2007:76(1):8-17. 
11. IRVINE E, WIILLAMS C. Treatment-, and Disease-Related Factors and the Emergence of Adverse Events with Tyrosine Kinase Inhibitors for the Treatment of Chronic Myeloid Leukemia. Pharmacotherapy, 2013:.33: 868-881.

12. LANEUVILLE P. When to Stop Tyrosine Kinase Inhibitors for the Treatment of Chronic Myeloid Leukemia. Curr. Treat. Options in Oncol, 2018: Acesso disponível em: <https://doi.org/10.1007/s11864-018-0532-2>.

13. SANTOS VLCG. Qualidade de vida de adultos com câncer colorretal com e sem ostomia. Rev Latinoam Enferm,. 2004, 12(6):875-83.

14. ROSS DM. et al. Chronic myeloid IEukaemia and tyrosine kinase inhibitor therapy: assessment and management of cardiovascular risk factors. Intern Med J, 2018: 48: 5-13.

15. 1SEIDL EMF, ZANNON CMLC. Qualidade de vida e saúde:aspectos conceituais e metodológicos. Cad Saúde Pública, 2004: .20(2) :973-88.

16. SILVA JAG. INCA Estimativa 2018: incidência de câncer no Brasil / Instituto Nacional de Câncer. Coordenação de Prevenção e Vigilância. - Rio de Janeiro: INCA, 2017.Estimativa de Câncer no Brasil 2016-2017.

17. SILVA ABA, et al. Acompanhamento farmacoterapêutico em leucemia mieloide crônica: avaliação das intervenções farmacêuticas. Boletim Informativo Geum, 2016: 7(1) p:. 82-92.

18. SILVEIRA CA, FERRARI I. First-line treatment with imatinib mesylate in patients with chonic phase chronic myeloid leukemia: experience of a public hospital in develpoing country of South America. Leuk Lymphoma, 2012:53(7):14171419.

19. TERRA FS, et al. Avaliação da qualidade de vida de pacientes oncológicos submetidos à quimioterapia. Rev Bras Clin Med., 2013: 11(2):112-117.

20. USUKI K, et al. Efficacy and safety of nilotinib in Japanese patients with imatinib-resistant or -intolerant $P h+C M L$ or relapsed/refractory $\mathrm{Ph}+\mathrm{ALL}$ : a 36-month analysis of a phase I and II study. International Journal of Hematology,2012: 95 ( 4): 409-419.

21. WORLD HEALTH ORGANIZATION. World Cancer Report, 2008. Lyon: International Agency for Research on Cancer; 2009.

22. VARDIMAN JW. et al. The 2008 revision of the World Health Organization (WHO) classification of the myeloid neoplasms and acute leukemia. Blood, 2009; 114(5): 937-951.

23. VENA C. et al. Sleep-wake disturbances in people with cancer part II: evaluating the evidence for clinical decision making. Oncol Nurs Forum, 2004:31(4):747-767.

24. YU CH, et al. Factors associated with the quality of life of family caregivers for leukemia patients Health and Quality of Life Outcomes, $2017: 15(55), 2-11$. 